\title{
Long-term body-weight supported treadmill training and subsequent follow-up in persons with chronic SCI: effects on functional walking ability and measures of subjective well-being
}

\author{
Spinal Cord (2006) 44, 265-266. doi:10.1038/sj.sc.3101827; published online 13 September 2005
}

Hicks et $a l^{1}$ reported on a clinical trial in which chronic incompletely paralyzed spinal cord-injured (SCI) patients have undergone a locomotor training on the treadmill with varying degrees of body weight support over a period of 12 months. The therapeutic interventions are similar to those we and others have been employing since the early 1990s (for a controlled trial see Wernig et $a l^{2}$ ); therefore, a closer comparison of the outcome and of methodical details is warranted. In both trials, several patients, wheelchair bound, nonambulating at the onset of therapy (class 0 in the modified or unmodified 'Wernig scale'), made considerable progress in their locomotor capabilities, some reaching independent walking. This fact alone is important in that it shows that locomotor training focusing on upright walking is quite effective even when started years after spinal cord damage. Thus, the principle derived from the pioneer work on spinal cats ${ }^{3}$ that even the (isolated) spinal cord is capable of (activity dependent) motor learning applies to incompletely, including near-completely, paralyzed SCI persons. The conclusion 'to intensely train walking if walking is to be relearned' is nearly self-evident. ${ }^{45}$ The treadmill and suspension system is an excellent and by far the most practical device to optimize such training without being exclusive (in fact, the first most severely paralyzed SCI person we saw walking over firm ground without help had trained himself without a treadmill (patient $Z^{5}$ ); remarkably, however, he had learned rules of spinal locomotion effective in spinal animals (summarized by Grillner, 1980)).

A marked difference in Hicks and our studies is in the number of patients who, after the specific therapy, had reached independent walking: while we saw 10 from 13 dependent (wheelchair bound) chronic patients with cervical or high thoracic lesions become independent (from functional classes 0-2 moving up to 3 and higher on our scale, see Figures 2 and 3 in Wernig et $a l^{2}$ ), there were only $1-2$ out of 10 in the trial by Hicks et al. ${ }^{1}$ Why the difference? Trying to find an answer one runs into several difficulties due to missing information: First we do not know the degree of paralysis in Hicks' patients since the authors choose the ASIA score (which is not made for this purpose). Manual testing of muscle activity in defined positions is mandatory, including the information in tetraplegics on the use of their arms for handling walking devices like rollator or canes. In addition, evoking multijoint movements in more dynamic settings including the treadmill needs to be done: flexion and extension patterns may be evokable, which can be trained and possibly utilized for walking over ground. ${ }^{6}$

Even more important is the question whether the authors trained their patients according to what we have termed 'rules of spinal locomotion'; only then are these motor programs evokable, if at all. Also, the amount of specific help provided by the therapist in moving the limbs is crucial: Therapists (and even more so robots moving the limbs with the patient suspended over the treadmill) have the tendency to move limbs passively without demanding that the patient activates all his residual voluntary activity; this can easily be tested when therapists suddenly stop their help with the treadmill still moving. Quite often the patient is doing much less than he is capable of with the effect that training is inadequate; in fact, recent animal experiments experiments indicate that the rigid support provided by robots is even worse than no support at all. ${ }^{7}$ As it stands, we have no information on training over firm ground in the study by Hicks et al. In our protocol, there is a test to walk over ground once a week and over ground walking is increasingly performed as soon as positive signs are noted. If training was performed on the treadmill only, the poor performance over ground would not be a surprise.

Thus, it would be beneficial for a just evaluation of this remarkable work that the authors supplied some of the missing information listed above. For future studies, it will be important to standardize training and assessments according to published protocols and clearly indicate deviations if performed (including the rationale for doing so). As a contribution along this line and possible basis for discussion, we have set into the Internet our detailed manual for training SCI and other neurological patients including stroke (www.meb.uni-bonn.de/wernig).

Hicks et al have modified our Functional Scale now including 10 rather than six classes. While the finer grading might be interesting for laboratory investigations, ours is more oriented according to practical 
aspects of walking in everyday life, it demands larger steps of progress and has a high reliability. ${ }^{6}$ In the data presented by Hicks et al, there seems little need to deviate from our simple scale.

A Wernig Department of Physiology II, University Bonn, Wilhelmstrasse 31, D-53111 Bonn, Germany

\section{References}

1 Hicks et al. Long-term body-weight supported treadmill training and subsequent followup in persons with chronic SCI: effects on functional walking ability and measures of subjective well-being. Spinal Cord 2005; 43: 291-298.

2 Wernig A, Muller S, Nanassy A, Cagol E. Laufband therapy based on 'rules of spinal locomotion' is effective in spinal cord injured persons. Eur J Neurosci 1995; 7: 823-829 (Erratum in: Eur J Neurosci 1995; 7:1429).

3 Lovely RG, Gregor RJ, Roy RR, Edgerton VR. Effects of training on the recovery of full-weight-bearing stepping in the adult spinal cat. Exp Neurol 1986; 92: 421-435.

4 Wernig A, Muller S. Improvement of walking in spinal cord injured persons after treadmill training. In: Wernig A (ed). Restorative Neurology. Vol. 5. Elsevier Science Publishers BV: Amsterdam 1991 pp 475-485.

5 Wernig A, Muller S. Laufband locomotion with body weight support improved walking in persons with severe spinal cord injuries. Paraplegia 1992; 30: 229-238.

6 Maegele M, Muller S, Wernig A, Edgerton VR, Harkema SJ. Recruitment of spinal motor pools during voluntary movements versus stepping after human spinal cord injury. J Neurotrauma 2002; 19: 1217-1229.

7 Cai LL et al. IEEE International Conference on Rehabilitation Robotics (ICORR) Chicago, USA, June 28-July 1, 2005 pp 575-579. 